\title{
Stable Sunspot Equilibria in a Cash-in-Advance Economy*
}

\author{
George W. Evans \\ University of Oregon
}

Seppo Honkapohja
University of Cambridge

Ramon Marimon

University of Pompeu Fabra, Barcelona

February 23, 2004; revised

\begin{abstract}
We analyze a monetary model with flexible labor supply, cash-inadvance constraints and seigniorage-financed government deficits. If the intertemporal elasticity of substitution of labor is greater than one, there are two steady states, one determinate and the other indeterminate. If the elasticity is less than one, there is a unique steady state, which can be indeterminate. Only in the latter case do there exist sunspot equilibria that are stable under adaptive learning. A sufficient reduction in government purchases can in many cases eliminate the sunspot equilibria while raising consumption/labor taxes even enough to balance the budget may fail to achieve determinacy.

JEL classifications: C62, D83, D84, E31, E32

Key words: Indeterminacy, learnability, expectational stability, endogenous fluctuations, seigniorage.

${ }^{*}$ Financial support from the US National Science Foundation Grant No. 0136848 and from grants by the Academy of Finland, Bank of Finland, Yrjö Jahnsson Foundation and Nokia Group is gratefully acknowledged. The research was to a large extent done while the second author was affiliated with the Research Unit on Economic Structures and Growth,
\end{abstract} University of Helsinki. 
Corresponding author: Professor George W. Evans, Department of Economics, 1285 University of Oregon, Eugene OR 97403-1285, USA. email: gevans@oregon.uoregon.edu

\section{Introduction}

There has been considerable interest in the issue of indeterminacy in both theoretical and applied macroeconomics. The distinctive feature of indeterminacy is that there are multiple well-behaved rational expectations (RE) solutions to the model. These can take various forms, including a dependence on extraneous random variables (called "sunspots"). Such solutions correspond to self-fulfilling prophecies and have been offered as an explanation of the business cycle. Much of this recent research has focused on stationary sunspot equilibria (SSEs) near an indeterminate steady state, a possibility that has been examined in both extensions of Real Business Cycle (RBC) models, incorporating externalities, and in a variety of monetary models. ${ }^{1} \mathrm{~A}$ detailed survey of this literature is provided by (Benhabib and Farmer 1999), which gives extensive references.

A crucial related issue is the learnability of such solutions. Suppose agents are not assumed to have rational expectations a priori but instead make forecasts using a perceived law of motion with parameters that they update over time using an adaptive learning rule, such as least squares. Can such learning rules lead agents eventually to coordinate on an SSE? That it is indeed possible for sunspot solutions to be learned by agents was demonstrated by (Woodford 1990) in the context of the Overlapping Generations (OG) model of money. (See also (Evans 1989)). Local stability conditions for Markov SSEs in simple forward looking models were developed in (Evans and Honkapohja 1994). ${ }^{2}$ In general it can be shown that the possibility of such coordination depends on stability conditions for the SSE, as will be discussed further below. It is also easy to develop examples in which SSEs are not stable under learning. For an extensive treatment of adaptive learn-

\footnotetext{
${ }^{1}$ In nonlinear models SSEs can also exist near multiple distinct steady states or rational deterministic cycles.

${ }^{2}$ Examples of learnable SSEs are the "growth cycles" studied in (Evans, Honkapohja, and Romer 1998) and the "animal spirits" equilibrium provided by (Howitt and McAfee 1992). These SSEs fluctuate between neighborhoods of two distinct steady states, so that nonlinearity of the model is a crucial element.
} 
ing in macroeconomics, see (Evans and Honkapohja 2001), who show that "expectational stability" (E-stability) conditions typically govern the local learnability of SSEs, as well as other RE solutions. ${ }^{3}$

Recent work has found that in RBC type models SSEs near an indeterminate steady state fail to be stable under learning; see Chapter 10 of (Evans and Honkapohja 2001), (Rudanko 2002) and (Evans and McGough 2002). This raises the question whether stable SSEs under learning could exist in dynamic representative agent market-clearing models with a unique steady state. In this paper we demonstrate that this is indeed possible using a standard infinite-horizon monetary general equilibrium model. ${ }^{4}$

We study a model where the money demand arises from a cash-in-advance constraint, as in (Svensson 1985). Svensson's cash-in-advance timing requires that consumption must be purchased with existing money holdings and, therefore, any unexpected rise of the price level results in consumption losses that could have been avoided if agents had the possibility of adjusting their portfolios within the purchasing period, as in Lucas's cash-in-advance timing. ${ }^{5}$ For this reason, the model studied here is now being widely used in monetary economics. ${ }^{6}$ In these models, the price elasticity of money demand corresponds to the labor supply elasticity of intertemporal substitution. To assume an elasticity of intertemporal substitution greater than or equal to one is common in RBC models. However, microeconomic estimates of this elasticity are typically significantly smaller than one, in the range 0.5 to 0.05 ; see (Card 1994) for a survey of the microeconometric literature.

Our paper contributes to the monetary literature by providing a characterization of the set of equilibria in a cash in advance model with seigniorage, stressing the role played by the elasticity of intertemporal substitution and, in particular, by studying the stability properties of the different equilibria under adaptive learning. Our findings are both sharp and somewhat unex-

\footnotetext{
${ }^{3}$ In the context of one-step forward-looking univariate models, results showing the existence of stable SSE in the neigborhood of a steady state are given in (Evans and Honkapohja 2003b) and (Evans and Honkapohja 2003c).

${ }^{4}$ Recently, it been shown that stable SSEs can exist in the sticky price New Keynesian models for specific interest rate rules; see (Honkapohja and Mitra 2001) and (Evans and McGough 2003).

${ }^{5}$ See (Woodford 1994) for an extensive discussion of indeterminacy in cash-in-advance models with Lucas's timing.

${ }^{6}$ For example, (Nicolini 1998) shows that, if the monetary authority maximizes the welfare of the representative agent, and agents have isoelastic preferences, then the Ramsey policy is time inconsistent unless the price elasticity is one (the $\log$ case).
} 
pected. The model has two regimes and the results depend on the regime. In the first, characterized by an elasticity of intertemporal substitution greater than one, there are two steady states and a continuum of rational expectations equilibria (REE) with a long-run inflation corresponding to the high inflation steady state. The low inflation steady state is determinate and stable under learning, while the other is indeterminate but unstable under learning. In this regime, none of the SSEs near the indeterminate steady state are learnable.

In contrast, when the elasticity of intertemporal substitution is less than one, which is consistent with micro evidence, there is a unique steady state that can be either determinate or indeterminate. When the elasticity is sufficiently low, the steady state is indeterminate and there are nearby Markov chain sunspot equilibria that are stable under learning. In a neighborhood of this steady state, there also exist stationary autoregressive (AR) solutions, depending on extraneous sunspot noise, but these are not stable under learning. These results show that requiring stability under learning redirects the focus of analysis to a particular type of indeterminate steady state and to particular SSEs near that steady state. The results also show that the choice of the elasticity of intertemporal substitution has important consequences in terms of the nature of the underlying equilibria. ${ }^{7}$

When stable SSEs do exist, this raises the issue of whether economic policy can be used to avoid them, either by eliminating the existence of SSEs or by rendering them unstable. In our model, where government finances expenditures through seigniorage, the natural policy variable is the level of such purchases. We find that for a wide range of parameter values in which the steady state has nearby stable Markov sunspot equilibria, lowering government purchases sufficiently can render the steady state determinate, making the nearby sunspot solutions disappear.

The above results all concern the case where consumption and labor taxes are ignored. However, in Section 6 we show how the analysis can be extended to taxes. Most of the results concerning the effects of changing government spending remain unchanged when tax rates are low; in a wide range of cases a sufficient reduction in government spending will yield determinacy. We also consider the possibility of eliminating learnable sunspot equilibria by

\footnotetext{
${ }^{7}$ We remark that versions of the OG model with seigniorage can also lead to the two types of regimes studied here. However, as is well known, the case of a unique indeterminate steady state in the OG model corresponds to a perverse labour supply response.
} 
increases in consumption and labor taxes. Here the results are more subtle. If government spending is not too high, raising consumption and labor taxes can be sufficient to eliminate indeterminacy and learnable sunspots. However, if government spending is relatively high then even raising taxes sufficiently to achieve budget balance will fail to eradicate learnable sunspot equilibria.

\section{The Model}

We consider an infinite-horizon representative agent economy. There are two types of consumption goods, cash and credit goods. (Cash goods must be paid for by cash at hand.) There is also a flexible labor supply, one unit of which produces one unit of either consumption good. The unit endowment of time is split between leisure and labor. Both consumption goods are perishable and there are no capital goods.

Let the utility function be

$$
U_{t}=E_{t} \sum_{s=t}^{\infty} \mathfrak{B}^{s-t}\left[\frac{\left(c_{s}^{1}\right)^{1-\sigma}}{1-\sigma}+\frac{\left(c_{s}^{2}\right)^{1-\sigma}}{1-\sigma}+\alpha \frac{\left(1-n_{s}\right)^{1-\sigma}}{1-\sigma}\right]
$$

where $c_{s}^{1}, c_{s}^{2}$ and $n_{s}$ denote cash goods, credit goods and labor supply, respectively. $\mathfrak{B}$ is the discount factor and $\sigma>0$ the coefficient of relative risk aversion. That is, $1 / \sigma$ is the elasticity of intertemporal substitution. The (sub-)utilities of cash goods, credit goods and leisure are assumed identical to facilitate investigation and presentation of the results. $\alpha$ is the relative weight placed on leisure.

The household budget constraint is

$$
M_{s+1}+B_{s+1} \leq p_{s}\left(n_{s}-c_{s}^{1}-c_{s}^{2}\right)+M_{s}+I_{s} B_{s} .
$$

Here $M_{s+1}$ and $B_{s+1}$ denote the stocks of money and bonds at the beginning of period $s+1$. $I_{s}$ is the nominal one-period interest rate factor on risk-free bonds earned during period $s$ and known at the end of period $s-1 . p_{s}$ is the price of goods and labor in period $s$. The cash-in-advance constraint (CA constraint) takes the form ${ }^{8}$

$$
p_{s} c_{s}^{1} \leq M_{s}
$$

\footnotetext{
${ }^{8}$ This is the Svensson timing, while Lucas's timing would had been $p_{s} c_{s}^{1} \leq M_{s+1}$.
} 
We will focus on equilibria where bonds are not held. Defining

$$
m_{s+1}=M_{s+1} / p_{s} \text { and } \pi_{s}=p_{s} / p_{s-1},
$$

we can write the first order conditions as

$$
\begin{aligned}
\left(c_{t}^{2}\right)^{-\sigma} & =\mathfrak{B} E_{t}^{*}\left[\pi_{t+1}^{-1}\left(c_{t+1}^{1}\right)^{-\sigma}\right] \\
\left(c_{t}^{2}\right)^{-\sigma} & =\mathfrak{B} E_{t}^{*}\left[I_{t+1} \pi_{t+1}^{-1}\left(c_{t+1}^{2}\right)^{-\sigma}\right] \\
\left(c_{t}^{2}\right)^{-\sigma} & =\alpha\left(1-n_{t}\right)^{-\sigma} .
\end{aligned}
$$

Here $E_{t}^{*}$ denotes the expectations of the household, conditional on time $t$ information, where we use the notation $E_{t}^{*}$ to indicate that the expectations are not necessarily assumed to be fully rational, due to adaptive learning. When RE are assumed we will use the notation $E_{t}$. With the CA constraint holding with equality, it can be written as

$$
c_{t}^{1}=m_{t} / \pi_{t}
$$

The market clearing condition is

$$
n_{t}=c_{t}^{1}+c_{t}^{2}+g_{t}
$$

where $g_{t}$ denotes government spending on goods. We assume that $g_{t}$ is an iid random variable with small bounded support around the mean $g>0$. There is also a government finance constraint taking the form

$$
B_{t+1}+M_{t+1}=p_{t} g_{t}+I_{t} B_{t}+M_{t} .
$$

Note that for simplicity we are ignoring taxes at this stage. We generalize the model to include consumption and labor taxes in Section 6. If bonds are not held in positive net amount in equilibrium, then this constraint yields the familiar seigniorage equation

$$
\pi_{t}=\frac{m_{t}}{m_{t+1}-g_{t}} .
$$

Household optimization, market clearing and the CA constraint lead to the equations

$$
\begin{aligned}
n_{t} & =1-\alpha^{1 / \sigma} \mathfrak{B}^{-1 / \sigma}\left\{E_{t}^{*}\left[\pi_{t+1}^{-1}\left(c_{t+1}^{1}\right)^{-\sigma}\right]\right\}^{-1 / \sigma} \\
m_{t+1} & =\left(1+\alpha^{-1 / \sigma}\right) n_{t}-\alpha^{-1 / \sigma} \\
c_{t}^{1} & =m_{t+1}-g_{t} \\
c_{t}^{2} & =n_{t}-c_{t}^{1}-g_{t}, \\
I_{t+1} & =\left(c_{t}^{2}\right)^{-\sigma} \mathfrak{B}^{-1}\left\{E_{t}^{*}\left[\pi_{t+1}^{-1}\left(c_{t+1}^{1}\right)^{-\sigma}\right]\right\}^{-1}
\end{aligned}
$$


Note that $m_{t+1}=M_{t+1} / p_{t}$, the real money stock carried forward from period $t$ to period $t+1$, is determined at time $t$. Similarly, $I_{t+1}$ is determined and known in period $t$. Equations (3)-(7), together with (2), give the temporary equilibrium equations determining $\pi_{t}, n_{t}, m_{t+1}, c_{t}^{1}, c_{t}^{2}$ and $I_{t+1}$ as functions of time $t$ expectations, the exogenous government spending shock $g_{t}$, and the previous period's real money stock $m_{t}$.

We note that the labor supply response in this model is entirely standard. It can be shown that, under perfect foresight, dynamic labor supply is characterized by

$$
\frac{1-n_{t}}{1-n_{t+1}}=\mathfrak{B} R_{t+1}^{-1 / \sigma}
$$

where $R_{t+1}=I_{t+1} / \pi_{t+1}$. Thus increases in the real interest rate factor $R_{t+1}$ lead to increases in current labor supply $n_{t}$ for any value of $\sigma$.

\section{Linearized Model}

Our first step is to determine the possible steady states and then to loglinearize the model around the steady states. ${ }^{9}$ This will enable us to examine determinacy and stability under learning through an extension of the procedure developed in (Evans and Honkapohja 2003c).

\subsection{Nonstochastic Steady States}

We begin by determining the non-stochastic perfect foresight steady states that are possible when $g_{t}=g$ is constant and nonstochastic. Denoting steady state values by bars over the variables, (3)-(5) and (2) imply

$$
\begin{aligned}
\bar{n} & =1-\alpha^{1 / \sigma} \mathfrak{B}^{-1 / \sigma} \bar{\pi}^{1 / \sigma} \bar{c}^{1} \\
\bar{m} & =\left(1+\alpha^{-1 / \sigma}\right) \bar{n}-\alpha^{-1 / \sigma} \\
\bar{c}^{1} & =\bar{m}-g \\
\bar{\pi} & =\bar{m} /(\bar{m}-g) .
\end{aligned}
$$

These equations can be reduced to a single equation in the steady state inflation rate $\bar{\pi}$,

$$
(1-g) \bar{\pi}=1+g A \bar{\pi}^{1 / \sigma},
$$

\footnotetext{
${ }^{9}$ When the shocks are sufficiently small, determinacy and stability under learning of stochastic equilibria can be examined through linearization. See Chapters 11-12 of (Evans and Honkapohja 2001) for this methodology in basic nonlinear forward-looking models.
} 


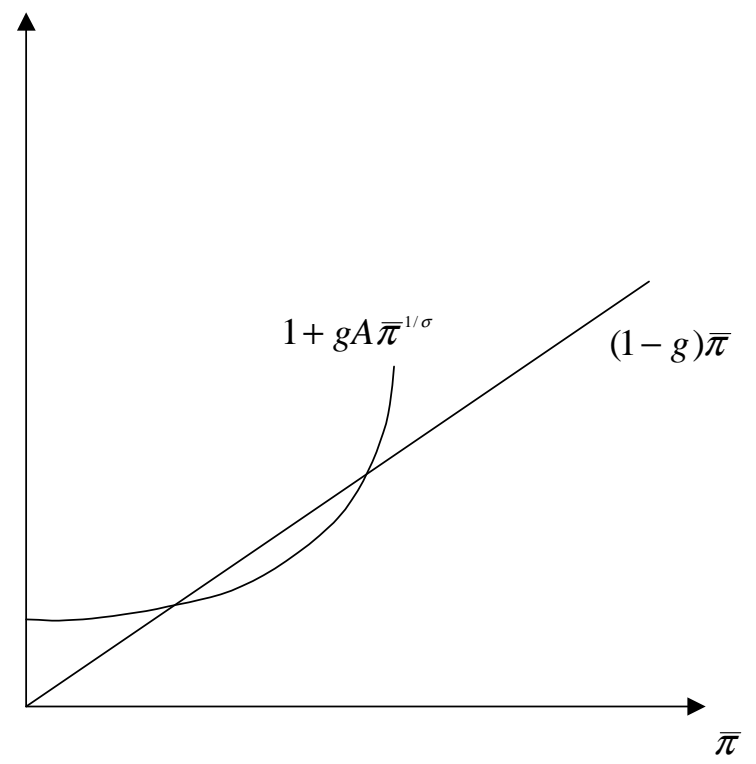

Figure 1: $\sigma<1$

where $A=\left(1+\alpha^{1 / \sigma}\right) \mathfrak{B}^{-1 / \sigma}>1$ since $\alpha>0$ and $\mathfrak{B}<1$.

For $g=0$ there is a unique steady state $\pi=1$. For $g>0$, it can be seen that the model has two regimes, depending on $\sigma$. If $\sigma<1$, then the right-hand side of (8) is a convex function, while the left-hand side defines a straight line and there are two cases. If $g>0$ is below a threshold value, depending on $\alpha, \sigma$ and $\mathfrak{B}$, there are two distinct steady states, $1<\bar{\pi}_{L}<\bar{\pi}_{H}$, while if $g$ exceeds this threshold there are no perfect foresight steady states. Below this threshold value, increases in $g$ raise $\bar{\pi}_{L}$ and lower $\bar{\pi}_{H}$. The $\sigma<1$ regime is standard in seigniorage models. However, this model also has a less familiar regime that arises when $\sigma>1$. In this case the right hand side of (8) is concave, and provided $0<g<1$ (which we assume throughout the paper), there is a unique steady state $\bar{\pi}$. In this regime increases in $g$ raise $\bar{\pi}$. Figures 1 and 2 illustrate the two cases $\sigma<1$ and $\sigma>1$.

\subsection{Linearization}

We begin by remarking that, in the stochastic economy, agents are assumed to make forecasts $E_{t}^{*}\left[\pi_{t+1}^{-1}\left(c_{t+1}^{1}\right)^{-\sigma}\right]$ about a nonlinear function of future inflation 


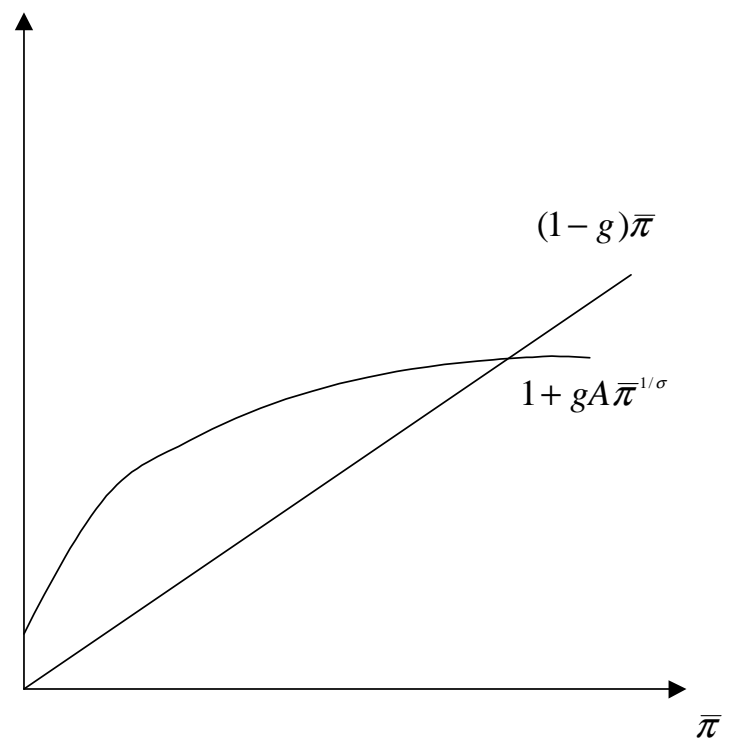

Figure 2: $\sigma>1$

and consumption of the cash good, see equation (3). When the model is loglinearized for analysis of indeterminacy and learning, we must decide on the formulation that will be used and there are two natural possibilities. First, agents might forecast separately the two variables in the linearization of the composite quantity $\pi_{t+1}^{-1}\left(c_{t+1}^{1}\right)^{-\sigma}$. Under this assumption the model can be reduced to a bivariate system in inflation and consumption of the cash good (or equivalently employment). Second, it is possible to derive a univariate reduced form from (2)-(7) and conduct the analysis with the univariate form. In what follows, we adopt the latter formulation for theoretical simplicity. Not surprisingly, both formulations deliver identical results. ${ }^{10}$

Using (1), (2), (4) and (5) we can write (3) in the form

$$
c_{t}^{1}=1-A\left\{E_{t}^{*}\left[\frac{\left(c_{t+1}^{1}\right)^{1-\sigma}}{c_{t}^{1}+g_{t}}\right]\right\}^{-1 / \sigma}-g_{t},
$$

where $A=\left(1+\alpha^{1 / \sigma}\right) \mathfrak{B}^{-1 / \sigma}$ is as in equation (8). To obtain this equation, we have in effect assumed that agents understand, and when forecasting

\footnotetext{
${ }^{10}$ The results for the bivariate formulation are available on request.
} 
make use of, the CA and seigniorage relations (1)-(2). ${ }^{11}(9)$ is the univariate formulation that we will employ. Let $g_{t}=g+\hat{u}_{t}$, where $\hat{u}_{t}$ is now assumed to be white noise with mean zero.

The log-linearization of (9) is

$$
z_{t}=\beta_{0} E_{t}^{*} z_{t}+\beta_{1} E_{t}^{*} z_{t+1}+u_{t}
$$

where we have introduced the notation $z_{t}=\log c_{t}^{1}-\log \bar{c}^{1}$ and

$$
\begin{aligned}
\beta_{0} & =-\frac{A}{\sigma}(\bar{\pi})^{(1-\sigma) / \sigma}, \\
\beta_{1} & =\frac{A(1-\sigma)}{\sigma}(\bar{\pi})^{1 / \sigma} \text { and } \\
u_{t} & =-\frac{g}{\bar{c}^{1}} \hat{u}_{t} .
\end{aligned}
$$

Note that $\beta_{0}<0$ while $\beta_{1} \gtrless 0 \Leftrightarrow \sigma \lessgtr 1$. We make the regularity assumptions that $\beta_{0} \neq 1, \beta_{1} \neq 0$ and $\beta_{0}+\beta_{1} \neq 1$. In deriving (10) we have made the assumption that current exogenous, but not current endogenous variables, are known when expectations are formed. ${ }^{12}$ This strikes us as the most natural assumption within the univariate framework, but we will comment on an alternative assumption below. (Under RE, knowledge of the exogenous and predetermined variables is equivalent to knowledge of all endogenous variables, but this equivalence does not hold under learning.)

We next assess the determinacy of the steady state and consider the different types of REE using the linearized model (10).

\subsection{Noisy Steady State and AR Solutions}

The method of undetermined coefficients can be used to show that the loglinearized reduced form (10) has RE solutions taking the form of "noisy steady states,"

$$
z_{t}=\left(1-\beta_{0}\right)^{-1} u_{t}
$$

These solutions are often called minimal state variable (MSV) solutions in the terminology of (McCallum 1983) and are the solutions most typically adopted in applied work.

\footnotetext{
${ }^{11}$ Again, these assumptions are made for theoretical simplicity. They are not imposed in our later numerical simulations of the multivariate nonlinear model under learning.

${ }^{12}$ Knowledge of aggregate $c_{t}^{1}$ is equivalent to knowlege of the current price level and inflation rate.
} 
If the steady state, around which we have linearized the model, is determinate then, as is well known, this is the unique stationary solution near the steady state. See, for example, (Blanchard and Kahn 1980), (Farmer 1999) and (Evans and Honkapohja 2001). On the other hand, if the steady state is indeterminate there exist SSEs in a neighborhood of that steady state. ${ }^{13}$ The possibility of modeling business cycle fluctuations as SSEs has been emphasized by (Cass and Shell 1983), (Azariadis 1981), (Farmer 1999) and (Guesnerie and Woodford 1992). The following result is easily verified:

Proposition 1 The general linear model (10) is indeterminate if and only if

$$
\left|\frac{\beta_{1}}{1-\beta_{0}}\right|>1 \text {. }
$$

This follows since, under RE, (10) is equivalent to the standard one-step forward-looking model $z_{t}=\beta_{1}\left(1-\beta_{0}\right)^{-1}\left(E_{t} z_{t+1}+u_{t}\right)$ studied e.g. in (Farmer 1999). Some intuition for the indeterminacy condition in the Proposition is provided by noting that it requires strong feedback from expectations to the current value of the endogenous variable. As is well known, strong expectational feedbacks are needed for the existence of self-fulfilling prophecies.

A familiar feature of the seigniorage model is that in the case of two steady states the high inflation equilibrium is indeterminate. As we will see in Section 4.2, this result also holds in our model. In addition, it can be shown that for some parameter values we have indeterminacy in the regime $\sigma>1$ when there is a single steady state. In fact we have:

Corollary 2 In the case $\sigma>1$ the steady state in the monetary model is indeterminate when $\sigma$ is sufficiently large.

This result can be seen as follows. First note from (8) that $\lim _{\sigma \rightarrow \infty} \bar{\pi}=$ $(1+2 g) /(1-g)$, implying that $\lim _{\sigma \rightarrow \infty} \bar{c}^{1}=(1-g) / 3$. Second, we have $\lim _{\sigma \rightarrow \infty} \beta_{0}=0$ and $\lim _{\sigma \rightarrow \infty} \beta_{1}=-2$. With these values the condition in Proposition 1 is satisfied.

In the case of Figure 1, the two steady states $\bar{\pi}_{H}$ and $\bar{\pi}_{L}$ have the following determinacy properties:

\footnotetext{
${ }^{13}$ The terminology "regular" and "irregular" is often used synonymously with "determinate" and "indeterminate."
} 
Corollary 3 In the case $\sigma<1$ the high inflation steady state $\bar{\pi}_{H}$ is indeterminate and the low inflation steady state $\bar{\pi}_{L}$ is determinate.

The result is proved in the Appendix.

We next consider the form of SSEs in cases of indeterminate steady states. Using the method of undetermined coefficients one can show that there exist stochastically stationary solutions of the form

$$
z_{t}=a+b z_{t-1}+d_{0} u_{t}+d_{1} u_{t-1}+k \eta_{t}
$$

where $\eta_{t}$, the sunspot variable, is an arbitrary (observable) exogenous variable satisfying $E_{t} \eta_{t+1}=0$. Computing conditional expectations under (12) and substituting into (10), it is seen that the REE of the form (12) consist of the steady state (with $a=b=d_{1}=k=0$ and $d_{0}=\left(1-\beta_{0}\right)^{-1}$ ) or a continuum with $a=0, b=\left(1-\beta_{0}\right) / \beta_{1}, d_{1}=-\beta_{1}^{-1}$ and $d_{0}, k$ free. For convenience we will refer to the latter as autoregressive (AR) SSEs, though they also include moving average dependencies on the intrinsic disturbance. This is the type of solution that is emphasized by much of the applied indeterminacy literature, see e.g. (Benhabib and Farmer 1999). For the case at hand, when the steady state is indeterminate, there are stationary AR SSEs. Stationarity stipulates that $|b|=\left|\left(1-\beta_{0}\right) / \beta_{1}\right|<1$, which holds precisely when the steady state is indeterminate, see Proposition 1.

Although the AR solutions are the form of SSEs that have recently received the most attention, the literature has also drawn attention to the existence of solutions generated by finite state Markov solutions. We next show that this type of solution can exist in our monetary model.

\subsection{Markov Sunspot Solutions}

When a steady state is indeterminate it follows from the theoretical literature that there will also exist SSEs around the steady state for which the sunspot process is a Markov chain with a finite number of states; see (Chiappori, Geoffard, and Guesnerie 1992) for the case without intrinsic shocks. We will call such solutions Markov SSEs to distinguish them from the AR SSEs discussed above. (Evans and Honkapohja 2003c) examine the relation between these two types of SSEs in the basic one-step forward looking model without intrinsic shocks.

For simplicity, we focus on SSEs driven by a 2-state Markov chain. Thus assume that $s_{t}$ is a two state exogenous process, taking values $s_{t}=1$ or 
$s_{t}=2$. The transition probabilities are $p_{i j}, j=1,2$, so that $p_{12}=1-p_{11}$ and $p_{21}=1-p_{22}$. Let $P=\left(p_{i j}\right)$. We look for solutions of the form:

$$
z_{t}=z(j)+K u_{t} \text { if } s_{t}=j .
$$

To satisfy (10) under RE the values of $z(j)$ must satisfy

$$
z(j)=\frac{\beta_{1}}{1-\beta_{0}}\left[p_{j 1} z(1)+p_{j 2} z(2)\right], \text { and } K=\left(1-\beta_{0}\right)^{-1},
$$

of which the first equation can be rewritten in the vector form

$$
\theta=\mathcal{T} \theta, \text { where } \theta^{\prime}=(z(1), z(2)), \mathcal{T}(\theta)=\frac{\beta_{1}}{1-\beta_{0}} P
$$

A Markov SSE $\theta$ exists if there exist $0<p_{11}<1$ and $0<p_{22}<1$ and $\theta \neq 0$ for which $\theta$ satisfies the equation (13). Note that if an SSE exists then, in our linearized model, $k \theta$ is also an SSE for any real $k$ for the same transition probabilities, so that the "size" of the sunspot fluctuations is indeterminate. Formally, SSEs exist if and only if $\mathcal{T}-I$ is singular for some $0<p_{11}, p_{22}<1$. Noting that $\mathcal{T}$ depends on $p_{11}$ and $p_{22}$, we can solve the equation

$$
\operatorname{det}\left(\mathcal{T}\left(p_{11}, p_{22}\right)\right)=0
$$

and make use of the fact that $P$ is a 2 -dimensional transition probability matrix. In linearized models such as the current one, SSEs exist only for very particular transition probabilities, namely those for which $\frac{\beta_{1}}{1-\beta_{0}}$ is the inverse of an eigenvalue of $P$. Since $P$ has one eigenvalue equal to 1 and the other in the open interval $(-1,1)$, when $\left|\frac{\beta_{1}}{1-\beta_{0}}\right|>1$, i.e. the steady state is indeterminate, we always have existence of Markov SSEs. The required condition on $P$ can be thought of as a resonant frequency condition that makes possible the excitation of the SSE. ${ }^{14}$

\section{$4 \quad$ Stability Under Learning}

We now take up the question of stability of the RE solutions under adaptive learning rules. In the case of indeterminate steady states we separately assess

\footnotetext{
${ }^{14}$ For other transition probabilities the matrix $\mathcal{T}$ is nonsingular and the equation $\theta=\mathcal{T} \theta$ has only the trivial solution $\theta=0$, which corresponds to the steady state.
} 
each of the three types of solution for their stability under learning. Least squares and related learning dynamics have been widely studied and shown to converge to the usually employed REE in many standard models. This is true of the stationary solutions of, for example, the Cagan model of inflation, the Sargent-Wallace IS-LM-PC model, the Samuelson overlapping generations model and the real business cycle model. A recent overview of the literature is provided in (Evans and Honkapohja 2001).

The starting point for analysis of learning is the temporary equilibrium in the model, given by equation (9) or its linearization (10). To complete the description of the agents' behavior we must supplement this equation with a rule for forecasting the required state variables next period. The agents are assumed to have perceptions about the (in general stochastic) equilibrium process of the economy. This is usually called the perceived law of motion (PLM) and depends on parameters that are updated as new data become available over time. At each period $t$, agents form expectations by making forecasts using the estimated PLM. This leads to a temporary equilibrium, called the actual law of motion (ALM), which provides the agents a new data point of the key variables. Estimated parameters are updated in each period according to least squares and the new data. The issue of interest is the stability under learning of some rational expectations solution, i.e. whether the estimated parameters of the PLM converge to REE values over time.

It is well known that, for a wide range of models, stability under least squares learning is governed by E-stability conditions. See the (Evans and Honkapohja 2001) book for an extensive discussion of these concepts and analytical techniques. The E-stability conditions are developed as follows. For given values of the parameters of the PLM one computes the resulting ALM, and E-stability is then determined by a differential equation in notional time in which the parameters adjust in the direction of the ALM parameter values.

We provide E-stability conditions for the different REE in the general linear model (10). These results extend (Evans and Honkapohja 2003c) to models with intrinsic shocks and by including the $E_{t}^{*} z_{t}$ term, which arises under our information assumptions. We remark that the stability results in our linearized monetary model are unaffected if instead full $t$-dating of expectations is assumed. 


\subsection{Steady State and AR Solutions}

Consider the noisy steady state solutions (11). These solutions exist whether or not a steady state is indeterminate. We outline the technique for determining stability under learning for this simple case. For other cases details are relegated to the Appendix.

Agents have a PLM of the form

$$
z_{t}=a+K u_{t}
$$

Note that, like the steady state REE, the PLM is a linear function of the shock and it naturally incorporates an intercept since log-linearization around the steady state was done only for analytical purposes. Under adaptive learning agents are assumed to estimate $a$ and $K$ by recursive least squares.

Given estimates $a_{t}$ and $K_{t}$ in period $t$, the temporary equilibrium is then given by (10) with $E_{t}^{*} z_{t}=a_{t}+K_{t} u_{t}$ and $E_{t}^{*} z_{t+1}=a_{t}$. The question of interest is whether $a_{t} \rightarrow \bar{a}, K_{t} \rightarrow \bar{K}$ as $t \rightarrow \infty$, where $\bar{a}=0, \bar{K}=\left(1-\beta_{0}\right)^{-1}$ is the REE. The answer is that convergence is governed by E-stability.

To determine E-stability one assumes expectations $E_{t}^{*} z_{t}=a+K u_{t}$, $E_{t}^{*} z_{t+1}=a$, based on the above PLM, for an arbitrary $a$ and $K$ (intuitively, $a_{t}$ and $K_{t}$ evolve asymptotically slowly under adaptive learning). Substituting these into (10) the implied ALM takes the form

$$
z_{t}=\left(\beta_{0}+\beta_{1}\right) a+\left(\beta_{0} K+1\right) u_{t}
$$

This gives rise to a mapping $T(a, K)=\left(\left(\beta_{0}+\beta_{1}\right) a, 1+\beta_{0} K\right)$, and E-stability is defined as the local asymptotic stability of the fixed point $(\bar{a}, \bar{K})$ of the differential equations

$$
\frac{d a}{d \tau}=\left(\beta_{0}+\beta_{1}\right) a-a, \frac{d K}{d \tau}=1+\beta_{0} K-K
$$

where $\tau$ is virtual or notional time. (Note that this is simply a partial adjustment formula in the virtual time.) Clearly, the general stability conditions for (14) are $\beta_{0}+\beta_{1}<1$ and $\beta_{0}<1$.

Consider next the AR SSEs. Agents are assumed to have the PLM (12) and to make forecasts based on the estimated PLM. E-stability conditions are derived in the Appendix. Collecting the results together, we have: 
Proposition 4 For the general linear model (10) we have:

(i) the E-stability condition for the noisy steady state is

$$
\beta_{0}+\beta_{1}<1 \text { and } \beta_{0}<1
$$

(ii) necessary conditions for E-stability of the AR SSEs are

$$
\beta_{1}<0 \text { and } \beta_{0}>1
$$

Intuition for the steady-state E-stability condition (i) is fairly straightforward. Under this PLM, agents are estimated the mean of the process. If $\beta_{0}+\beta_{1}>1$ then estimates of the mean that are higher than the steady state value will be revised upward, i.e. away from the equilibrium value. In essence too much positive feedback from expectations to current endogenous data destabilizes the system. The additional requirement $\beta_{0}<1$ is needed because the PLM also depends on the exogenous shock $u_{t}$. We omit the more elaborate intuition that could be developed for (ii).

We remark that the E-stability condition in (i) is not the same as the determinacy condition. The condition in (i) of Proposition 4 can be examined numerically for different values of the parameters in the monetary model. Recalling that $\beta_{0}<0$ and that $\sigma>1$ implies $\beta_{1}<0$, we have:

Corollary 5 For the linearized monetary model if $\sigma>1$ the unique steady state is E-stable irrespective of its determinacy/indeterminacy.

When $\sigma<1$ and there are two steady states we have:

Corollary 6 For the linearized monetary model if $\sigma<1$ the steady state $\bar{\pi}_{L}$ is E-stable and the steady state $\bar{\pi}_{H}$ is not E-stable.

This result follows directly from the observation that $\beta_{0}+\beta_{1}>1$ at $\bar{\pi}_{H}$ and $\beta_{0}+\beta_{1}<1$ at $\bar{\pi}_{L}$, which is shown in the proof of Corollary 3 in the Appendix.

Using part (ii) of Proposition 4, we get:

Corollary 7 The AR SSEs are not E-stable in the linearized monetary model.

We remark that in related models, the AR paths can in some cases be Estable. See, for example, the monetary OG model of (Duffy 1994). 


\subsection{Markov Solutions}

Finally, we consider the Markov SSEs. Suppose that in addition to the shock $u_{t}$, agents observe a sunspot $s_{t}$ satisfying the resonant frequency condition and that they consider also conditioning their actions on the values of the sunspot. A simple learning rule is that agents run least squares regressions on the sunspot state and $u_{t}$. Agents then make forecasts using these estimates and the transition probabilities for the observed sunspot (these probabilities can also be estimated if they are unknown). ${ }^{15}$ The stability of SSEs under adaptive learning depends upon the corresponding E-stability condition and we now examine E-stability of the Markov SSEs. In the Appendix we discuss the definition of E-stability for this set-up. The results are:

Proposition 8 The E-stability conditions for Markov SSEs in the general linear model (10) are $\beta_{0}<1$ and $\beta_{0}+\beta_{1}<1$.

We remark that the E-stability conditions for the Markov SSEs are identical to those for steady states and the intuition is analogous. The key feature of E-stable Markov SSEs is that they arise in the neighborhood of a indeterminate steady state that is stable under steady state learning. The phenomenon of SSEs inheriting E-stability properties of nearby steady-state solutions arises more generally, see (Evans and Honkapohja 1994) and Chapter 12 of (Evans and Honkapohja 2001).

Corollary 9 In the linearized monetary model we have: (i) if $\sigma \geq 1$ and the single steady state $\bar{\pi}$ is indeterminate, the Markov SSEs are E-stable and (ii) if $\sigma<1$ the Markov SSEs near an indeterminate steady state are not E-stable.

Part (i) of Corollary 9 follows at once by noting that $\sigma \geq 1$ implies $\beta_{1} \leq 0$ while always $\beta_{0}<0$. Part (ii) follows from noting that $\sigma<1$ implies $\beta_{1}>0$ and then indeterminacy requires $\beta_{0}+\beta_{1}>1$, which contradicts the E-stability condition in Proposition 8.

\footnotetext{
${ }^{15}$ In terms of the original nonlinear model, an alternative learning procedure would be for agents to directly estimate the mean of $\pi_{t+1}^{-1}\left(c_{t+1}^{1}\right)^{-\sigma}$ conditional on the current sunspot state $s_{t}$. In the simulations below we adopt this alternative scheme when examining the nonlinear model numerically. The stability properties of the two approaches appear to be identical.
} 
We now give a numerical example of a Markov SSE satisfying the resonant frequency condition in the linearized model.

Example of a Markov SSE: Suppose that $\mathfrak{B}=0.95, \alpha=1, \sigma=3.5$ and $g=.19 .{ }^{16}$ The steady state is indeterminate: $\beta_{1} /\left(1-\beta_{0}\right)=-1.2408$. There are Markov SSEs with, for example, $p_{11}=0.1041$ and $p_{22}=0.09$, and these SSEs are stable under learning.

Two important remarks must be made at this point. First, it is possible for a steady state to be stable under learning and at the same time for nearby SSEs to be stable under learning. When this occurs, the solution to which the economy converges depends on the form of the PLM, i.e. on whether or not agents in their learning allow for a possible dependence on sunspots.

Second, the results of this section are subject to the qualification that our linearized monetary model has been derived from an underlying nonlinear model. Therefore, we investigate the existence and stability of SSEs directly for the original nonlinear model using numerical computations and simulations when $\sigma \geq 1$.

There are several important differences between the nonlinear system (2)(5) and the linearized system (10) with respect to the Markov SSEs. In the linearized system the resonant frequency condition (equation (21) in the Appendix) must be satisfied exactly and the "size" of the SSE is indeterminate, as earlier discussed. In the exact nonlinear system there are Markov SSEs for transition probabilities close to the resonant frequency condition and the value of equilibrium is in part determined by these probabilities. This issue is fully analyzed for the univariate forward looking model in (Evans and Honkapohja 2003b). Thus, although the linearized model is convenient for obtaining existence and stability conditions for Markov SSEs, it is important to establish further details using the nonlinear model. ${ }^{17}$

Consider, therefore, the learning dynamics in the original nonlinear model (2)-(5). The key variable that agents must forecast is $X_{t+1}=\pi_{t+1}^{-1}\left(c_{t+1}^{1}\right)^{-\sigma}$.

\footnotetext{
${ }^{16}$ We have not tried to obtain the parameter values by calibrating the model to data, since our main goal is to provide numerical illustrations.

${ }^{17}$ We remark that, with $\sigma>1$, indeterminacy of the steady state does not imply the existence of deterministic cycles, which is in contrast to the monetary OG models analysed e.g. by (Grandmont 1985) and (Azariadis and Guesnerie 1986). The numerical example above does not have 2-cycles. Thus these SSEs are not local to pure cycles. However, pure cycles exist for some other parameter configurations (details are available on request).
} 
Because the sunspot variable is assumed to be first-order Markov, the conditional expectation of this variable depends only on the current state. A simple learning rule is thus to estimate the mean value of $X_{t+1}$ conditional on the current sunspot state at $t$, e.g. by state contingent averaging:

$$
\hat{X}_{i, t}=\left(\# N_{i}(t)\right)^{-1} \sum_{\substack{1 \leq \ell \leq t, s_{t-\ell-1}=i}} X_{t-\ell},
$$

$i=1,2$, where $\# N_{i}(t)$ denotes the number of data points in which $s_{t-\ell-1}=i$ for $1 \leq \ell \leq t$. Thus agents at time $t$ estimate the mean value that $X_{t+1}$ will take, next period, as $\hat{X}_{i, t}$, when the sunspot in period $t$ is in state $s_{t}=i$, $i=1,2{ }^{18}$ Accordingly they form expectations at $t$ as $E_{t}^{*} X_{t+1}=\hat{X}_{i, t}$. Over time the estimates $\hat{X}_{i, t}$ are revised in accordance with observed values of $X_{t}$ following each of the two different states.

Under adaptive learning the model consists of these learning dynamics together with the equations (2)-(7). We remark that under this formulation the realized value of $X_{t}$ depends on both $E_{t}^{*} X_{t+1}$ and $E_{t-1}^{*} X_{t}$ because $\pi_{t}$ depends on both $m_{t+1}$ and $m_{t}$. From the numerical results for the linearized model, we anticipate convergence to a stationary sunspot solution, for transition probabilities close to satisfying the resonant frequency condition, when $\sigma>1$ and the steady state is indeterminate.

We have simulated the nonlinear system and the corresponding E-stability differential equation using the parameter values from the above example. Figure 3 illustrates convergence to the sunspot equilibrium for the choice of transition probabilities $p_{11}=0.07$ and $p_{22}=0.05$ and initial conditions near the steady state. (The vertical axis shows deviations of $\hat{X}_{1}$ and $\hat{X}_{2}$ from the steady state value of $\bar{X}$ and the initial deviations were $\hat{X}_{1}=\bar{X}+0.01$ and $\left.\hat{X}_{2}=\bar{X}-0.01\right)$. The simulation clearly shows convergence to a Markov SSE. In this SSE the ratio of output in the two states is $n_{1} / n_{2}=1.11$ and expected inflation $E_{t}^{*} \pi_{t+1}$ in the two states are 1.20 and 2.86.

The results of this section show that, in the case in which $\sigma>1$ and the steady state is indeterminate, endogenous fluctuations due to expectational indeterminacy are a real concern. For exogenous sunspots near the resonant frequency, rational expectation SSEs exist and are stable under simple learning rules. These considerations raise the question of whether policy is able to avoid such expectational volatility.

\footnotetext{
${ }^{18}$ Because in the nonlinear model agents at time $t$ make only one-step ahead forecasts of $X_{t+1}$, it is unnecessary to directly estimate the dependence of $X_{t}$ on $g_{t}$.
} 


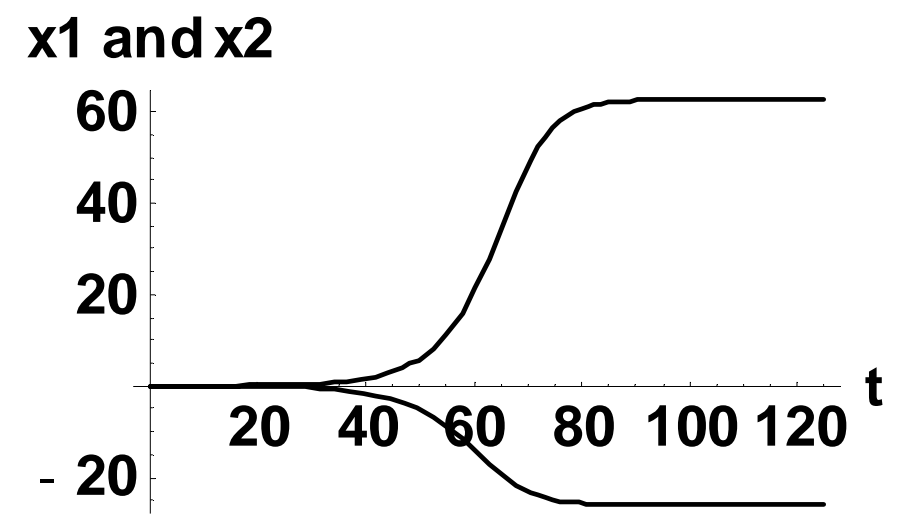

Figure 3: Convergence of adaptive learning to sunspot solution

\section{Changes in Policy}

We have seen that in this model there are two steady states, $\bar{\pi}_{L}$ and $\bar{\pi}_{H}$ when $\sigma<1$ and $g>0$. There has been much discussion of the issue of whether the economy might converge to the indeterminate steady state $\bar{\pi}_{H}$ in related seigniorage models. While we do take seriously the potential economic instability of the economy due to the multiplicity of equilibria in this case, we believe that the primary concern in this case is divergent paths (with $\pi_{t}$ increasing beyond $\bar{\pi}_{H}$ to unsustainable levels) if for some reason $\pi_{t}$ escapes from the basin of attraction of $\bar{\pi}_{L}$. Reductions in $g$ tend to stabilize the economy in this case, making convergence to $\bar{\pi}_{L}$ more likely. Fiscal constraints on deficits and debt can also play an important role. The stability results of this and other papers suggest that $\bar{\pi}_{H}$ and SSEs near $\bar{\pi}_{H}$ are not locally stable under learning, though divergent paths are a concern. ${ }^{19}$

However, a new case appears in our model of seigniorage finance. In the case $\sigma>1$ there is a unique steady state that can be determinate or indeterminate and for $\sigma$ sufficiently large it will necessarily be indeterminate. For values of $\sigma>1$ the (noisy) steady state is stable under steady state learning. Furthermore, if the steady state is indeterminate and agents condition their actions on an exogenous sunspot near the resonant frequency, then they

\footnotetext{
${ }^{19}$ The standard seigniorage model under learning was first studied by (Marcet and Sargent 1989). The role of fiscal constraints is discussed in (Evans, Honkapohja, and Marimon 2001).
} 
will converge to a noisy Markov SSE. Can policy help avoid the endogenous fluctuations which can arise in this case? ${ }^{20}$

That this is indeed possible is shown by the following result (see the Appendix for a proof): ${ }^{21}$

Proposition 10 Assume that $\sigma>1$ and consider the determinacy of the unique steady state $\bar{\pi}$ for different values of $g$.

(i) If $\sigma<2$, there exists a critical value $\bar{g}>0$ such that the steady state $\bar{\pi}$ is determinate if $g<\bar{g}$ and indeterminate if $g>\bar{g}$.

(ii) If $\left(1+\alpha^{1 / \sigma}\right) \mathfrak{B}^{-1 / \sigma}(\sigma-2)>\sigma$, the steady state $\bar{\pi}$ is indeterminate for all $g>0$.

By Corollary 9, part (i) of Proposition 10 also implies that E-stable Markov SSEs are eliminated by sufficient reductions in government spending $g$. Note that if $\alpha \geq 1$ then for $\sigma \geq 4$ all positive values of $g$ are consistent with stable Markov SSEs.

The results of Proposition 10 are illustrated in Table 1. We set $\alpha=1$ and fix the discount rate at $\mathfrak{B}=0.95$. For each value of $\sigma$ we examine the implications of different choices of $g$. Table 1 shows that for a substantial range of values for $\sigma$, endogenous fluctuations depending on extraneous sunspot variables can be avoided by decreasing government purchases $g$ sufficiently.

TABLE 1: Critical values of $g$ for $\alpha=1$.

\begin{tabular}{|c|c|c|c|c|c|c|c|c|c|}
\hline$\sigma$ & 1.1 & 1.5 & 2.0 & 2.5 & 3.0 & 3.5 & 3.7 & 3.9 & $\geq 4$ \\
\hline$g$ & 0.359 & 0.341 & 0.267 & 0.190 & 0.118 & 0.053 & 0.029 & 0.006 & 0 \\
\hline $\bar{\pi}$ & 16.89 & 4.39 & 2.56 & 1.84 & 1.44 & 1.175 & 1.091 & 1.018 & 1 \\
\hline
\end{tabular}

For each value of $\sigma$ reported in Table 1, a critical value of $g$ is reported, together with the associated value of the steady state inflation rate. At the stated or larger values of $g$ the steady state is indeterminate, there exist Markov SSEs, and these SSEs are stable under adaptive learning. For lower values of $g$ the steady state becomes determinate and SSEs near the steady

\footnotetext{
${ }^{20}$ Avoidance of endogenous fluctuations is a major concern in the design of interest-rate rules for monetary policy; see (Evans and Honkapohja 2003a) for a survey.

${ }^{21}$ These results are similar in spirit, but different in detail, from those obtained for the monetary OG model by (Grandmont 1986). The latter emphasizes policy to eliminate cycles, and thus the SSEs that are associated with cycles in the OG model.
} 
state no longer exist. For any $\sigma$ in the range $1<\sigma<4$ stable Markov SSEs exist for high values of $g$ but not for sufficiently low values. In this region a reduction in $g$ can bring about a double benefit by both reducing steady state inflation and eliminating SSEs. ${ }^{22}$

In the model of this paper we have focussed on the seigniorage case in which government purchases are financed entirely by printing money. Seigniorage models have been used most commonly as a potential explanation of hyperinflation, and our numerical example indeed emphasized the possibility of endogenous fluctuations arising at high levels of inflation when the level of seigniorage is large. It is immediate from Table 1, however, that stable Markov SSEs near indeterminate steady states can also arise at low levels of inflation. We have made no attempt to calibrate the model to actual economies, and this would only be more appropriate with more elaborate versions of the model. However, the theoretical results of this paper show that monetary models of this type indeed have the power to explain business cycle fluctuations.

\section{Extension: The Model with Taxes}

In this section we show that the introduction of consumption and labor taxes, while it raises revenues to finance government expenditures, does not change the qualitative features of the previous analysis, although it introduces non trivial effects of fiscal policy changes to the stability and learnability of equilibria.

Let $\tau_{s}^{c}$ and $\tau_{s}^{n}$ be the consumption and labor tax rates in period $s$. The household budget constraint becomes

$$
M_{s+1}+B_{s+1} \leq M_{s}+I_{s} B_{s}+p_{s}\left(1-\tau_{s}^{n}\right) n_{s}-p_{s}\left(1+\tau_{s}^{c}\right)\left(c_{s}^{1}+c_{s}^{2}\right)
$$

and the cash-in-advance constraint (CA constraint) takes the form

$$
p_{s}\left(1+\tau_{s}^{c}\right) c_{s}^{1} \leq M_{s}
$$

The effective marginal tax rate on labor income, $\tau$, is the relevant tax for our analysis. This is the fraction of additional labor income taken away,

\footnotetext{
${ }^{22}$ These results are quantitatively, but not qualitatively, sensitive to the choice of $\alpha$. For example, for $\alpha<1$ determinacy can be obtained, with sufficiently low $g$, for values of $\sigma$ less than an upper limit that now exceeds 4 .
} 
through taxes -in terms of effective consumption- holding savings fixed, and is given by

$$
\tau_{t}=\frac{\tau_{t}^{n}+\tau_{t}^{c}}{1+\tau_{t}^{c}} ; \text { i.e., }\left(1-\tau_{t}\right)=\frac{1-\tau_{t}^{n}}{1+\tau_{t}^{c}}
$$

Keeping the same notation as before, let

$$
\begin{aligned}
m_{s+1} & =M_{s+1} / p_{s}\left(1+\tau_{s}^{c}\right), b_{s+1}=B_{s+1} / p_{s}\left(1+\tau_{s}^{c}\right), \\
\pi_{s} & =p_{s} / p_{s-1}, \text { and } v_{s}=\left(1+\tau_{s}^{c}\right) /\left(1+\tau_{s-1}^{c}\right)
\end{aligned}
$$

We can now rewrite the household budget constraint as

$$
m_{s+1}+b_{s+1} \leq m_{s} \pi_{s}^{-1} v_{s}^{-1}+I_{s} b_{s} \pi_{s}^{-1} v_{s}^{-1}+\left(1-\tau_{s}\right) n_{s}-\left(c_{s}^{1}+c_{s}^{2}\right)
$$

and the Cash-in-Advance constraint as

$$
c_{s}^{1}=m_{t} \pi_{s}^{-1} v_{s}^{-1}
$$

The first order conditions of the household's maximization problem become

$$
\begin{aligned}
\left(c_{t}^{2}\right)^{-\sigma} & =\mathfrak{B} E_{t}^{*}\left[\pi_{t+1}^{-1} v_{t+1}^{-1}\left(c_{t+1}^{1}\right)^{-\sigma}\right] \\
\left(c_{t}^{2}\right)^{-\sigma} & =\mathfrak{B} E_{t}^{*}\left[I_{t+1} \pi_{t+1}^{-1} v_{t+1}^{-1}\left(c_{t+1}^{2}\right)^{-\sigma}\right] \\
\left(c_{t}^{2}\right)^{-\sigma} & =\alpha\left(1-\tau_{t}\right)^{-1}\left(1-n_{t}\right)^{-\sigma} .
\end{aligned}
$$

Similarly, the government finance constraint is

$$
M_{s+1}+B_{s+1} \geq M_{s}+I_{s} B_{s}+p_{s} g_{s}-p_{s} \tau_{s}^{n} n_{s}-p_{s} \tau_{s}^{c}\left(c_{s}^{1}+c_{s}^{2}\right) .
$$

Using $c_{s}^{1}+c_{s}^{2}+g_{s}=n_{s}$, this can be expressed as

$$
m_{s+1}+b_{s+1} \geq m_{s} \pi_{s}^{-1} v_{s}^{-1}+I_{s} b_{s} \pi_{s}^{-1} v_{s}^{-1}+d_{s},
$$

where

$$
d_{s}=g_{s}-\tau_{s} n_{s}
$$

is the period $s$ fiscal deficit. As before, if bonds are not held in positive net amount in equilibrium, then this constraint yields the following modified seigniorage equation

$$
\pi_{t}=\frac{m_{t}}{m_{t+1}-d_{t}} v_{t}^{-1} .
$$


Household optimization, market clearing and the CA constraint lead now to the equations

$$
\begin{aligned}
n_{t} & =1-\alpha^{1 / \sigma}\left(1-\tau_{t}\right)^{-1 / \sigma} \mathfrak{B}^{-1 / \sigma}\left\{E_{t}^{*}\left[\pi_{t+1}^{-1} v_{t+1}^{-1}\left(c_{t+1}^{1}\right)^{-\sigma}\right]\right\}^{-1 / \sigma} \\
m_{t+1} & =\left[\left(1-\tau_{t}\right)+\alpha^{-1 / \sigma}\left(1-\tau_{t}\right)^{1 / \sigma}\right] n_{t}-\alpha^{-1 / \sigma}\left(1-\tau_{t}\right)^{1 / \sigma} \\
c_{t}^{1} & =m_{t+1}-d_{t} \\
c_{t}^{2} & =n_{t}-c_{t}^{1}-g_{t} \\
I_{t+1} & =\left(c_{t}^{2}\right)^{-\sigma} \mathfrak{B}^{-1}\left\{E_{t}^{*}\left[\pi_{t+1}^{-1} v_{t+1}^{-1}\left(c_{t+1}^{1}\right)^{-\sigma}\right]\right\}^{-1}
\end{aligned}
$$

\subsection{Analysis}

The non-stochastic perfect foresight steady states are a generalization of the model without taxes. We set expenditures and tax rates to be deterministic constants, i.e., $g_{t}=g, v_{t}=1$ and $\tau_{t}=\tau$. Steady states are determined by the following equations,

$$
\begin{aligned}
\bar{n} & =1-\alpha^{1 / \sigma}(1-\tau)^{-1 / \sigma} \mathfrak{B}^{-1 / \sigma} \bar{\pi}^{1 / \sigma} \bar{c}^{1} \\
\bar{m} & =\left[(1-\tau)+\alpha^{-1 / \sigma}(1-\tau)^{1 / \sigma}\right] \bar{n}-\alpha^{-1 / \sigma}(1-\tau)^{1 / \sigma} \\
\bar{d} & =g-\tau \bar{n} \\
\bar{c}^{1} & =\bar{m}-\bar{d} \\
\bar{\pi} & =\bar{m} /(\bar{m}-\bar{d}) .
\end{aligned}
$$

These equations can be reduced to a single equation in the steady state inflation rate $\bar{\pi}$,

$$
(1-g) \bar{\pi}=1-\tau+\left[(g-\tau)+g \alpha^{1 / \sigma}(1-\tau)^{(\sigma-1) / \sigma}\right] \mathfrak{B}^{-1 / \sigma} \bar{\pi}^{1 / \sigma} .
$$

The earlier analysis clearly applies when $\tau$ is sufficiently small.

More generally, it is seen that there are two steady states when $\sigma<1$ and $\tau<g$. In contrast, for $\sigma>1$ there is always a unique steady state for all values of $\tau$. This follows from (19) since then the right-hand side is a concave function of $\bar{\pi}$ if the quantity in square brackets is positive and a decreasing function of $\bar{\pi}$ otherwise. The comparative statics with respect to $g$ are as before. For changes in the tax rate $\tau$, the comparative statics are clear-cut when $\sigma>1$ : increases in $\tau$ will reduce the unique steady state inflation rate. However, for $\sigma<1$ the comparative statics are in general ambiguous. 
To analyze determinacy and stability under learning, we require the full dynamic system. Assuming that tax rates are constant over time at rate $\tau_{s}=\tau$, the equations (18), together with (15), (16) and (17) can be combined to yield a univariate reduced form $c_{t}^{1}=\mathcal{G}\left(E_{t}^{*} \mathcal{H}\left(c_{t}^{1}, c_{t+1}^{1}, g_{t}, g_{t+1}\right), g_{t}\right)$, which has a linearization taking the same form (10) as before, but with coefficients $\beta_{0}$ and $\beta_{1}$ that now also depend on $\tau$. Details are given in the Appendix.

The methods developed earlier can be applied to consider different types of questions concerning setting of taxes $\tau$. We focus on the case unique steady state $(\sigma>1)$, for which we earlier demonstrated that stable Markov sunspot equilibria can arise. When also $\sigma<2$ it was shown above that sufficient reductions in $g$ will eliminate these sunspot solutions. This raises the natural question whether the same strong result can be obtained by sufficiently big increases in taxes. In particular, will determinacy of the steady state obtain when taxes are set to achieve budgetary balance in the mean?

Before addressing this issue we must check that it is feasible to balance the budget by increases in $\tau$. This is indeed true when $\sigma>1$ and there is a unique choice of $\tau$ which achieves budget balance. Setting $\bar{d}=0$ implies $\bar{\pi}=1$ and substituting this value in (19) we obtain that the required tax rate $\tau^{b}$ satisfies

$$
\tau\left(\mathfrak{B}^{1 / \sigma}+1\right)=g\left[\mathfrak{B}^{1 / \sigma}+1+\alpha^{1 / \sigma}(1-\tau)^{(\sigma-1) / \sigma}\right],
$$

which clearly has a unique solution $0<\tau^{b}<1$ since $0<g<1$. The Appendix gives the values of $\beta_{0}$ and $\beta_{1}$ when $\tau=\tau^{b}$ and we study the question numerically.

TABLE 2: Critical values of $g$ in balanced budget case for $\alpha=1$.

\begin{tabular}{|c|c|c|c|c|c|c|c|c|c|}
\hline$\sigma$ & 1.1 & 1.5 & 2.0 & 2.5 & 3.0 & 3.5 & 3.7 & 3.9 & 4 \\
\hline$g$ & 0.712 & 0.735 & 0.678 & 0.564 & 0.400 & 0.198 & 0.111 & 0.022 & 0 \\
\hline
\end{tabular}

It turns out that the balanced budget requirement is insufficient to guarantee determinacy and avoidance of learnable sunspot solutions. For this balancedbudget case Table 2 gives the critical values $\bar{g}$ such that indeterminacy and existence of learnable sunspots occurs for $g>\bar{g}$. Comparing to Table 1, it is seen that the balanced-budget requirement does, however, raise the level of $g$ that is possible before running into indeterminacy with learnable sunspots.

The finding that imposing budget balance using distortionary taxes does not eliminate indeterminacy is in itself not new: (Schmitt-Grohe and Uribe 
1997) showed a similar result in the context of the standard Real Business Cycle model. However, sunspot solutions in the RBC model with taxes are almost never stable under learning, as shown by (Evans and McGough 2002) and (Rudanko 2002). Our novel and intriguing result is that stable sunspot equilibria under learning can emerge when the budget is balanced through distortionary taxes instead of a reduction in government spending.

\section{Conclusions}

Indeterminacy of equilibria has been a major issue in both business cycle analysis and monetary economics. Most of the applied research has examined the question of the existence of self-fulfilling fluctuations in a neighborhood of an indeterminate steady state. This paper has imposed the additional discipline of asking whether there exist RE solutions, of this type, that are stable under adaptive learning dynamics. If solutions are not learnable, they may well be just theoretical artifacts. On the other hand, if they are stable under learning dynamics, then agents could plausibly coordinate on such solutions. We remark that, in experiments, agents appear able to condition their expectations on sunspots if, as a group, they have previously experienced fluctuations driven by fundamentals. In such case, it is possible that even once the 'fundamental uncertainty' vanishes, belief driven fluctuations persist (see (Marimon, Spear, and Sunder 1993)).

We have examined these issues in the context of a standard infinite horizon representative agent framework in which money demand is generated by cash in advance constraints and exogenous government spending is financed by a mixture of taxes and seigniorage. The model allows for both cash and credit goods as well as for a flexible labor supply for which the response to increased interest rates is always positive irrespective of the degree of curvature of the utility functions.

We have shown that, for some regions of the parameter space, there do exist learnable sunspot equilibria in a neighborhood of an indeterminate steady state. The learnable SSEs take a particular form, driven by a sunspot process taking the form of a finite state Markov chain with transition probabilities close to the resonant frequency property. The other types of SSEs examined are not learnable. In our model, the case of learnable SSEs arises for the regime in which there is a single steady state.

In many cases an appropriate change in fiscal policy can eliminate stable 
sunspot equilibria by rendering the steady state determinate. Achieving this goal requires some care in the choice and magnitude of the fiscal instruments. In some cases simply eliminating the government deficit will be enough to eradicate sunspot solutions. In other cases balancing the budget is in itself inadequate and reductions in the level of government spending are required.

\section{A Appendix: Technical Details}

Proof of Corollary 3: By Proposition 1 we need to show that $\beta_{0}+\beta_{1}>1$ at $\bar{\pi}_{H}$ and $\beta_{0}+\beta_{1}<1$ at $\bar{\pi}_{L}$ when $\sigma<1$.

Using (8), we have at a steady state $\bar{\pi}$

$$
\beta_{0}+\beta_{1}-1=\frac{[(1-g) \bar{\pi}-1][(1-\sigma) \bar{\pi}-1]}{\sigma g \bar{\pi}}-1 .
$$

Thus, we consider the quadratic function

$$
f(\pi)=[(1-g) \pi-1][(1-\sigma) \pi-1]-\sigma g \pi .
$$

The equation $f(\pi)=0$ has two roots $\pi=1$ and $\tilde{\pi}=(1-\sigma)^{-1}(1-g)^{-1}$. Clearly, $\beta_{0}+\beta_{1} \gtrless 1$ when $\bar{\pi} \gtrless \tilde{\pi}$.

On the other hand, consider the value $\breve{\pi}$ at which the graph of the function $1+g A \pi^{1 / \sigma}$ has the tangent parallel to the straight line $(1-g) \pi$. (Note that the two functions are the right- and left-hand sides of (8), respectively.) $\breve{\pi}$ solves the equation

$$
\frac{g A}{\sigma} \pi^{1 / \sigma-1}=1-g
$$

If we require $\breve{\pi}$ to be also a steady state, we get $\breve{\pi}=\tilde{\pi}$ and $g$ is at a maximal value for which steady states exist under the restriction $\sigma<1$. When $g$ is reduced from the maximal level, the comparative static properties of $\bar{\pi}_{H}$ and $\bar{\pi}_{L}$ imply that $\bar{\pi}_{L}<\tilde{\pi}$ and $\bar{\pi}_{H}>\tilde{\pi}$ and thus $\beta_{0}+\beta_{1}>1$ at $\bar{\pi}_{H}$ and $\beta_{0}+\beta_{1}<1$ at $\bar{\pi}_{L}$.

Proof of Proposition 4: Part (i) was derived in the text. For part (ii) concerning the AR solutions one starts with PLM of the form (12) and computes that, under the PLM

$$
\begin{aligned}
E_{t}^{*} z_{t} & =a+b z_{t-1}+d_{0} u_{t}+d_{1} u_{t-1}+k \eta_{t} \\
E_{t}^{*} z_{t+1} & =a+b E_{t}^{*} z_{t}+d_{1} u_{t}
\end{aligned}
$$


where it is assumed that the current values $u_{t}, \eta_{t}$ of the exogenous shocks, but not the endogenous variable $z_{t}$ are in the information set for period $t$. Substituting these into the linear model (10) we have

$$
\begin{aligned}
z_{t}= & \left(\beta_{0}+\beta_{1} b\right)\left(a+b z_{t-1}+d_{0} u_{t}+d_{1} u_{t-1}+k \eta_{t}\right) \\
& +\beta_{1} a+\beta_{1} d_{1} u_{t}+u_{t} .
\end{aligned}
$$

This yields the mapping from PLM to ALM, which, component by component, is given by

$$
\begin{aligned}
a & \rightarrow \beta_{1} a+\left(\beta_{0}+\beta_{1} b\right) a \equiv T_{a}(a, b) \\
b & \rightarrow\left(\beta_{0}+\beta_{1} b\right) b \equiv T_{b}(b) \\
d_{0} & \rightarrow\left(\beta_{0}+\beta_{1} b\right) d_{0}+\beta_{1} d_{1}+1 \equiv T_{d 0}\left(b, d_{0}, d_{1}\right) \\
d_{1} & \rightarrow\left(\beta_{0}+\beta_{1} b\right) d_{1} \equiv T_{d 1}\left(b, d_{1}\right) \\
k & \rightarrow\left(\beta_{0}+\beta_{1} b\right) k \equiv T_{k}(b, k) .
\end{aligned}
$$

Looking first at possible fixed points we see that $b=0$ or $b=\left(1-\beta_{0}\right) / \beta_{1}$. If $b=0$, we have $k=d_{1}=0, a=0$ and $d_{0}=\left(1-\beta_{0}\right)^{-1}$. This corresponds to the noisy steady state and in the determinate case this is the unique stationary solution. If $b=\left(1-\beta_{0}\right) / \beta_{1}$ we get $d_{1}=\beta_{1}^{-1}, a=0$ while $k$ and $d_{0}$ can take any values, which is the indeterminate case. This latter class of solutions will be stationary if $\left|\left(1-\beta_{0}\right) / \beta_{1}\right|<1$.

Introducing the notation $T\left(a, b, d_{0}, d_{1}, k\right)=\left(T_{a}, T_{b}, T_{d 0}, T_{d 1}, T_{k}\right)$, for $\mathrm{E}$ stability one examines the local stability of

$$
\frac{d\left(a, b, d_{0}, d_{1}, k\right)}{d \tau}=T\left(a, b, d_{0}, d_{1}, k\right)-\left(a, b, d_{0}, d_{1}, k\right)
$$

at the REE of interest. In the indeterminate case we say that the solution class is E-stable if the dynamics of the E-stability differential equations converge to some member of the class from each nearby initial condition. For the indeterminate case the E-stability conditions are easily seen to be $\beta_{1}<0$ and $\beta_{0}>1$.

Proof of Proposition 8: The PLM of the agents is equivalent to

$$
z_{t}=z(j)+K u_{t} \text { if } s_{t}=j .
$$

Given the informational assumptions the ALM is

$$
\begin{aligned}
z(j)^{*} & =\beta_{0} z(j)+\beta_{1}\left[p_{j 1} z(1)+p_{j 2} z(2)\right], j=1,2, \\
K^{*} & =\beta_{0} K+1 .
\end{aligned}
$$


where $z(j)^{*}$ is the actual mean of $z_{t}$ and $K^{*}$ is the actual $u_{t}$ coefficient, under this PLM, if $s_{t}=j$. The $T$ map for $K$ is the same as in the case of steady state learning and the corresponding condition for E-stability is satisfied when $\beta_{0}<1$. The equations for $z(j)$ can be written in the matrix form

$$
\theta^{*}=T \theta, \text { where } \theta^{*}=\left(\begin{array}{c}
z(1)^{*} \\
z(2)^{*}
\end{array}\right), T=\beta_{0} I+\beta_{1} P \text { and } \theta=\left(\begin{array}{c}
z(1) \\
z(2)
\end{array}\right) .
$$

We remark that the $T$ map in (20) is different from $\mathcal{T}$ in (13), due to our information assumption for the system under learning. However, they have the same fixed points and thus a Markov SSE exists if and only if there are transition probabilities $0<p_{j j}<1, j=1,2$, that satisfy

$$
\frac{1-\beta_{0}}{\beta_{1}}=p_{11}+p_{22}-1 \text {. }
$$

The mapping from the PLM to the corresponding ALM is here linear and given by the matrix in (20). E-stability is determined by the stability of the differential equation

$$
d \theta / d \tau=T \theta-\theta .
$$

We need to compute the eigenvalues of $T-I=\left(\beta_{0}-1\right) I+\beta_{1} P$. If $\lambda$ is an eigenvalue of $T-I$ then we must have

$$
0=\operatorname{det}\left(\left(\beta_{0}-1\right) I+\beta_{1} P-\lambda I\right)=\beta_{1}^{2} \operatorname{det}\left(P-\frac{\lambda+1-\beta_{0}}{\beta_{1}} I\right)
$$

so that

$$
\frac{\lambda+1-\beta_{0}}{\beta_{1}}=1 \text { or } p_{11}+p_{22}-1 .
$$

In the latter case we get $\lambda=0$ while the former yields $\lambda=\beta_{0}+\beta_{1}-1$ implying the requirement $\beta_{0}+\beta_{1}<1$. We remark that $T-I$ always has a zero eigenvalue as a result of the resonant frequency condition (21) and there being a continuum of Markov due to the linearity of the model. The E-stability differential equation

$$
\frac{d \theta}{d \tau}=(T-I) \theta
$$

has a zero eigenvalue and negative eigenvalue if $\beta_{0}+\beta_{1}<1$ holds and (21) is satisfied. Stability of the set of Markov SSEs then follows from the mathematical Lemma in the Appendix of (Honkapohja and Mitra 2001). 
Proof of Proposition 10: If $\sigma>1$, we have $\beta_{1}<0$ while always $\beta_{0}<0$. Indeterminacy of the steady state requires $\beta_{0}-\beta_{1}>1$. In general, we have

$$
\beta_{0}-\beta_{1}=-\frac{A}{\sigma}(\bar{\pi})^{(1-\sigma) / \sigma}-\frac{A(1-\sigma)}{\sigma}(\bar{\pi})^{1 / \sigma}
$$

and, defining $H(\bar{\pi}) \equiv \beta_{0}-\beta_{1}$, we note that $H(\bar{\pi})$ is strictly increasing in $\bar{\pi}$ with

$$
\begin{aligned}
\lim _{\bar{\pi} \rightarrow \infty} H(\bar{\pi}) & =\infty \\
\lim _{\bar{\pi} \rightarrow 1} H(\bar{\pi}) & =\frac{A(2-\sigma)}{\sigma} .
\end{aligned}
$$

We couple these limits with the observations (a) $\bar{\pi} \rightarrow \infty$ as $g \rightarrow 1$ and (b) $\bar{\pi} \rightarrow 1$ as $g \rightarrow 0$, which follow from (8).

To prove part (i), we note that $\lim _{\bar{\pi} \rightarrow 1} H(\bar{\pi})<1$ if $\sigma<2$, which by continuity and $\lim _{\bar{\pi} \rightarrow \infty} H(\bar{\pi})=\infty$ imply the existence of $\bar{g}>0$ as required. Part (ii) follows by noting that $\lim _{\bar{\pi} \rightarrow 1} H(\bar{\pi})>1$ when $\left(1+\alpha^{1 / \sigma}\right) \mathfrak{B}^{-1 / \sigma}(\sigma-$ 2) $>\sigma$ since $A=\left(1+\alpha^{1 / \sigma}\right) \mathfrak{B}^{-1 / \sigma}$; see the definition after equation (8).

Details on the Model with Taxes: We first set $\tau_{t}=\tau$. The equilibrium equations (18), combined with (15) and (16), yield a univariate reduced form, which can be expressed either in terms of $c_{t}^{1}$ or $m_{t+1}$ since

$$
\left(c_{t}^{1}+g_{t}\right)(1-\tau+B(\tau))=m_{t+1}(1+B(\tau))+\tau B(\tau) .
$$

where $B(\tau)=\alpha^{-1 / \sigma}(1-\tau)^{1 / \sigma}$. In the case of taxes it is somewhat more convenient to express the reduced form in terms of $m_{t+1}$. Letting $\tilde{A}(\tau)=$ $\left(1+\alpha^{1 / \sigma}(1-\tau)^{(\sigma-1) / \sigma}\right) \mathfrak{B}^{-1 / \sigma}$, we obtain

$$
\begin{aligned}
m_{t+1}= & (1-\tau)+\tilde{A}(\tau) \times \\
& \left\{E_{t}^{*}\left[\frac{1}{m_{t+1}}\left(\frac{m_{t+2}(1+B(\tau))+\tau B(\tau)}{1-\tau+B(\tau)}-g_{t+1}\right)^{1-\sigma}\right]\right\}^{-1 / \sigma} .
\end{aligned}
$$

The log-linearized form $(22)$ is

$$
\tilde{m}_{t+1}=\beta_{0} E_{t}^{*} \tilde{m}_{t+1}+\beta_{1} E_{t}^{*} \tilde{m}_{t+2}+\gamma g_{t+1},
$$

where

$$
\begin{aligned}
& \beta_{0}=\sigma^{-1}[1-(1-\tau) / \bar{m}] \\
& \beta_{1}=-\frac{(1-\sigma)(\bar{m}-(1-\tau))(1+B(\tau))}{\sigma[-\bar{g}(1-\tau+B(\tau))+(\bar{m}(1+B(\tau))+\tau B(\tau))]}
\end{aligned}
$$


We remark that, for the reduced form $c_{t}^{1}=\mathcal{G}\left(E_{t}^{*} \mathcal{H}\left(c_{t}^{1}, c_{t+1}^{1}, g_{t}, g_{t+1}\right), g_{t}\right)$, the log-linearized equations for $c_{t}^{1}$ would have the same coefficients $\beta_{0}$ and $\beta_{1}$ on the expectation terms $E_{t}^{*} c_{t}^{1}$ and $E_{t}^{*} c_{t+1}^{1}$. Using the steady state relationships of Section 3.1, it can be verified the these reduce to our earlier results when $\tau=0$.

For non-stochastic steady states with budget balance, i.e. $\bar{d}=0$, it can be shown that

$$
\beta_{0}=-\frac{\tilde{A}(\tau)}{\sigma}, \beta_{1}=\frac{\tilde{A}(\tau)}{\sigma}\left[(1-\sigma) \frac{1+B(\tau)}{1-\tau+B(\tau)}\right]
$$

and the condition for indeterminacy and existence of stable sunspots is as above, i.e. $\beta_{0}-\beta_{1}>1$ when $\sigma>1$.

\section{References}

Azariadis, C. (1981): "Self-Fulfilling Prophecies," Journal of Economic Theory, 25, 380-396.

Azariadis, C., And R. Guesnerie (1986): "Sunspots and Cycles," Review of Economic Studies, 53, 725-737.

Barnett, W., J. Geweke, And K. Shell (eds.) (1989): Economic Complexity: Chaos, Sunspots, Bubbles, and Nonlinearity. Cambridge University Press, Cambridge.

Benhabib, J., and R. E. Farmer (1999): "Indeterminacy and Sunspots in Macroeconomics," in (Taylor and Woodford 1999), chap. 6, pp. 387-448.

Blanchard, O., and C. Kahn (1980): "The Solution of Linear Difference Models under Rational Expectations," Econometrica, 48, 1305-1311.

CARD, D. (1994): "Intertemporal Labor Supply: An Assessment," in (Sims 1994), chap. 2, pp. 49-78.

Cass, D., And K. Shell (1983): "Do Sunspots Matter?," Journal of Political Economy, 91, 193-227. 
Chiappori, P. A., P.-Y. Geoffard, and R. Guesnerie (1992): "Sunspot Fluctuations around a Steady State: The Case of Multidimensional, One-Step Forward Looking Economic Models," Econometrica, 60, $1097-1126$.

Duffy, J. (1994): "On Learning and the Nonuniqueness of Equilibrium in an Overlapping Generations Model with Fiat Money," Journal of Economic Theory, 64, 541-553.

Evans, G. W. (1989): "The Fragility of Sunspots and Bubbles," Journal of Monetary Economics, 23, 297-317.

Evans, G. W., and S. Honkapohja (1994): "On the Local Stability of Sunspot Equilibria under Adaptive Learning Rules," Journal of Economic Theory, 64, 142-161.

(2001): Learning and Expectations in Macroeconomics. Princeton University Press, Princeton, New Jersey.

_ (2003a): "Adaptive Learning and Monetary Policy Design," Journal of Money, Credit and Banking, forthcoming.

(2003b): "Existence of Adaptively Stable Sunspot Equilibria near an Indeterminate Steady State," Journal of Economic Theory, 111, 125134 .

(2003c): "Expectational Stability of Stationary Sunspot Equilibria in a Forward-looking Model," Journal of Economic Dynamics and Control, $28,171-181$.

Evans, G. W., S. Honkapohja, and R. Marimon (2001): "Convergence in Monetary Inflation Models with Heterogeneous Learning Rules," Macroeconomic Dynamics, 5, 1-31.

Evans, G. W., S. Honkapohja, and P. Romer (1998): "Growth Cycles," American Economic Review, 88, 495-515.

Evans, G. W., and B. McGough (2002): "Indeterminacy and the Stability Puzzle in Non-Convex Economies," University of oregon economics department working paper no. 2002-14. 
(2003): "Monetary Policy, Indeterminacy and Learning," mimeo.

FARmer, R. E. (1999): The Economics of Self-Fulfilling Prophecies, Second edition. MIT Press, Cambridge, Mass.

Grandmont, J.-M. (1985): "On Endogenous Competitive Business Cycles," Econometrica, 53, 995-1045.

- (1986): "Stabilizing Competitive Business Cycles," Journal of Economic Theory, 40, 57-76.

Guesnerie, R., And M. Woodford (1992): "Endogenous Fluctuations," in (Laffont 1992), chap. 6, pp. 289-412.

Honkapohja, S., And K. Mitra (2001): "Are Non-Fundamental Equilibria Learnable in Models of Monetary Policy?," Working paper, www.valt.helsinki.fi/RAKA/seppo.hmtl.

Howitt, P., And R. P. McAfee (1992): "Animal Spirits," American Economic Review, 82, 493-507.

LAffont, J.-J. (ed.) (1992): Advances in Economic Theory: Sixth World Congress. Volume 2. Cambridge University Press, Cambridge, UK.

Marcet, A., and T. J. Sargent (1989): "Convergence of Least Squares Learning and the Dynamic of Hyperinflation," in (Barnett, Geweke, and Shell 1989), pp. 119-137.

Marimon, R., S. E. Spear, and S. Sunder (1993): "Expectationally Driven Market Volatility: An Experimental Study," Journal of Economic Theory, 61, 74-103.

McCallum, B. (1983): "On Nonuniqueness in Linear Rational Expectations Models: An Attempt at Perspective," The Journal of Monetary Economics, 11, 139-168.

Nicolini, J. P. (1998): "More on the Time Inconsistency of Optimal Monetary Policy," Journal of Monetary Economics, 41, 333-350.

Rudanko, L. (2002): "On the Implications of a Balanced Budget Rule - An Adaptive Learning Perspective," Research report 93, University of Helsinki. 
Schmitt-Grohe, S., And M. Uribe (1997): "Balanced-Budget Rules, Discretionary Taxes and Aggregate Instability," Journal of Political Economy, 105, 976-1000.

Sims, C. (ed.) (1994): Advances in Econometrics, Sixth World Congress, Vol. 2. Cambridge University Press, Cambridge, UK.

Svensson, L. E. (1985): "Money and Asset Prices in a Cash-in-Advance Economy," Journal of Politcal Economy, 93, 919-944.

TAYlor, J., And M. Woodford (eds.) (1999): Handbook of Macroeconomics, Volume 1. Elsevier, Amsterdam.

Woodford, M. (1990): "Learning to Believe in Sunspots," Econometrica, $58,277-307$.

(1994): "Monetary Policy and Price Level Determinacy in a Cashin-Advance Economy," Economic Theory, 4, 345-380. 